\title{
The next generation: etravirine in the treatment of HIV-I infection in adults refractory to other antiretrovirals
}

This article was published in the following Dove Press journal:

Virus Adaptation and Treatment

8 July 2010

Number of times this article has been viewed

\author{
R Chris Rathbun \\ Michelle D Liedtke \\ Department of Clinical and \\ Administrative Sciences, College of \\ Pharmacy, University of Oklahoma \\ Health Sciences Center, Oklahoma \\ City, USA
}

\begin{abstract}
Etravirine is a second-generation nonnucleoside reverse transcriptase inhibitor (NNRTI) that is approved for the treatment of adult human immunodeficiency virus (HIV)infected patients with documented or suspected resistance to first-generation NNRTIs. Etravirine has a flexible molecular structure that allows it to retain its activity against mutant HIV strains that exhibit resistance to first-generation agents. It is evident that 3 or more etravirine resistance-associated mutations are typically necessary before clinical resistance to etravirine. Safety and efficacy of etravirine are established in antiretroviral treatment-experienced patients in combination with antiretroviral regimens that contain darunavir/ritonavir. In phase III studies, cutaneous reactions occurred in $19 \%$ of treated patients and are the most commonly observed adverse event. The typical manifestation is the development of a maculopapular rash within the first few weeks of etravirine therapy. Resolution commonly occurs within 1-2 weeks on continued therapy. Rare cases of severe skin reactions $(<0.1 \%)$ have been reported. Etravirine is hepatically metabolized by cytochrome P450 (CYP) 3A4, CYP2C9, and CYP2C19. Drug interactions with some antiretrovirals (eg, unboosted protease inhibitors and fosamprenavir/ ritonavir) and medications for other comorbidities (eg, atorvastatin and clarithromycin) have been reported and may require dosage adjustment for the coadministered drug or selection of alternative therapy in some instances. Administration of etravirine with potent inducers of CYP450 is not recommended due to the potential for subtherapeutic etravirine concentrations. In this article, the pharmacology, efficacy, safety, and tolerability of etravirine in adult treatmentexperienced patients with HIV-1 infection are reviewed. Medical literature was identified by MEDLINE and EMBASE searches (1966 to February 2010) using the search terms "etravirine", "TMC125", and "NNRTI".
\end{abstract}

Keywords: etravirine, TMC125, non-nucleotide reverse transcriptase inhibitor, antiretrovival, HIV

\section{Introduction}

It is estimated that 33.4 million people globally were living with human immunodeficiency virus (HIV) infection, with approximately 2.7 million new infections per year as of 2008. Two million deaths are attributed to HIV infection each year, 280,000 of which are in children younger than 15 years. ${ }^{1}$ Of the estimated 9.5 million HIV-infected individuals in developing and transitional countries, 42\% have access to antiretroviral therapy, resulting in declining rates of mortality due to the acquired immune deficiency syndrome (AIDS). ${ }^{1}$ In the United States, approximately 56,300 new infections occur each year. ${ }^{2}$ AIDS-related mortality has declined in the United States since 1996 with the introduction of highly active antiretroviral therapy. ${ }^{3}$ HIV infection is now managed as a chronic infection,
Correspondence: Michelle D Liedtke II I 0 N. Stonewall, CPB 229, Oklahoma City, OK 73II7, USA

Tel +I 405 27I 6878 ext 47260

Fax + I $40527 \mid 6430$

Email michelle-liedtke@ouhsc.edu 
requiring life-long antiretroviral therapy to improve and sustain immune function.

The use of combination therapy with 3 or more antiretrovirals represents the current standard of care for the treatment of HIV infection. Protease inhibitors and nonnucleoside reverse transcriptase inhibitors (NNRTIs) are presently recognized as preferred agents for treatment of patients without prior antiretroviral treatment experience. ${ }^{4-6}$ Integrase inhibitors have recently been added as another preferred option in some instances. ${ }^{4}$ Selection of protease inhibitor-, NNRTI-, or integrase inhibitor-based antiretroviral therapy is based on regimen potency, potential for resistance, drug interaction potential, and adherence considerations. In treatmentexperienced patients, selection of antiretrovirals is based on the presence of documented or suspected resistance. ${ }^{4-6}$
A summary of approved antiretrovirals and recommended antiretroviral regimens for treatment-naive patients is shown in Figure 1.

The NNRTIs were first introduced in 1996 and are widely used as initial therapy for therapy-naive patients due to their simplicity in dosing and overall favorable tolerability profile; however, they are susceptible to the rapid emergence of drug resistance during therapy, particularly if patients are nonadherent. ${ }^{7}$ In addition, de novo resistance to antiretroviral therapy has been observed in up to $20 \%-24 \%$ of patients, including first-generation NNRTIs (efavirenz, nevirapine, and delavirdine), before beginning treatment. ${ }^{8}$ Therefore, a significant need has existed to identify new NNRTI compounds that retain their activity in the presence of mutant HIV strains that harbor resistance to first-generation agents. The discovery

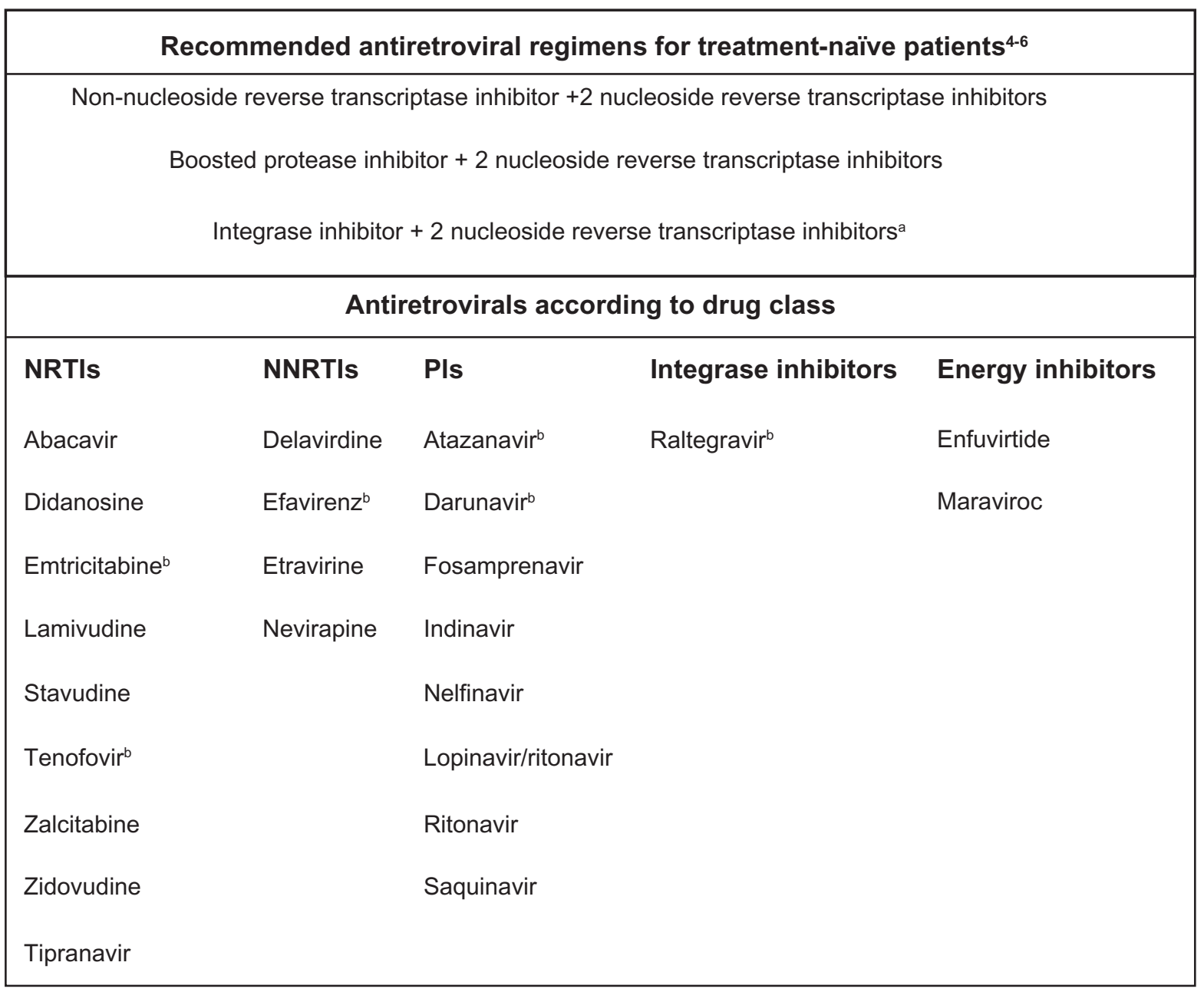

Figure I Antiretroviral therapy recommendations and list of approved agents.

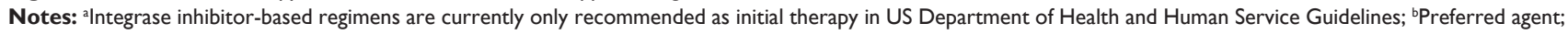
preferred protease inhibitors require coadministration with low-dose ritonavir.

Abbreviations: NRTIs, nucleoside reverse transcriptase inhibitors; NNRTls, nonnucleoside reverse transcriptase inhibitors; Pls, protease inhibitors. 
and synthesis of etravirine are the culmination of 15 years of exploration to identify compounds with activity against both wild-type and mutant HIV strains exhibiting resistance to early generation NNRTIs. ${ }^{9}$ Etravirine was approved for use in the United States and Europe in 2008 and represents the first second-generation NNRTI. This article reviews the pharmacology, efficacy, safety, and tolerability of etravirine in adult treatment-experienced patients with HIV-1 infection. Medical literature was identified by MEDLINE and EMBASE searches (1966 to February 2010) using the search terms "etravirine", "TMC125", and "NNRTI". Additional references were obtained from reference lists of published articles and by reviewing meeting abstracts. The manufacturer was also contacted to provide unpublished data.

\section{Pharmacology Mechanism of action}

HIV reverse transcriptase is responsible for the transcription of single-stranded viral RNA into single-stranded DNA, which is eventually integrated into the host DNA as double-stranded DNA. Like other NNRTIs, etravirine is a noncompetitive inhibitor of HIV reverse transcriptase and binds to subunit p66 of reverse transcriptase, resulting in a conformational change in the catalytic site for reverse transcriptase that limits its activity. ${ }^{10}$ Etravirine (formerly TMC125) is a diarylpyrimidine that has the chemical name 4-[(4-cyanophenyl)amino]4-pyrimidinyl]oxy]-3,5-dimethylbenzonitrile and chemical formula $\mathrm{C}_{20} \mathrm{H}_{15} \mathrm{BrN}_{6} \mathrm{O} .{ }^{11}$ Etravirine has a flexible molecular structure, allowing it to bind to multiple conformations in a hydrophobic binding pocket adjacent to the catalytic site of reverse transcriptase and retain its activity in the presence of mutations that confer resistance to first-generation NNRTIs. ${ }^{10,12}$ The structure of etravirine and other currently available NNRTIs is depicted in Figure 2.

Etravirine exhibits potent in vitro activity against wild-type HIV-1 strains, with median 50\% effective concentrations $\left(\mathrm{EC}_{50}\right)$ ranging from 1.4 to $4.8 \mathrm{nmol} / \mathrm{L}$ that are comparable to the activity of efavirenz $(1.2-3.4 \mathrm{nmol} / \mathrm{L}) .{ }^{11}$ Similar activity is observed against first-generation NNRTIresistant virus $\left(\mathrm{EC}_{50} \leq 10 \mathrm{nM}\right.$ for $83 \%$ of 6,171 recombinant clinical isolates tested) as well as non-clade B and circulating recombinant strains that are becoming more prevalent due to immigration and heterosexual transmission. ${ }^{11,13}$ Unlike other NNRTIs, etravirine also demonstrates weak activity against HIV-2 strains, with an $\mathrm{EC}_{50}$ of $3.5 \mu \mathrm{M}$; however, this is not believed to be clinically relevant. ${ }^{11}$

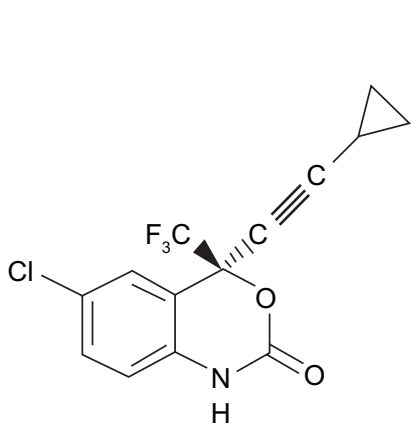

Efavirenz

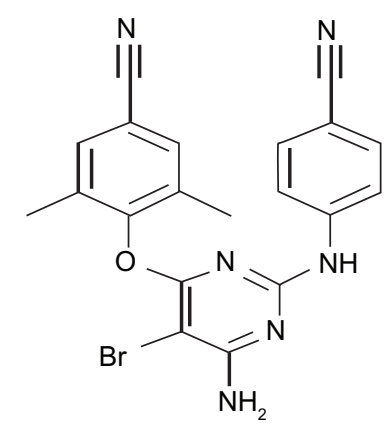

Etravirine

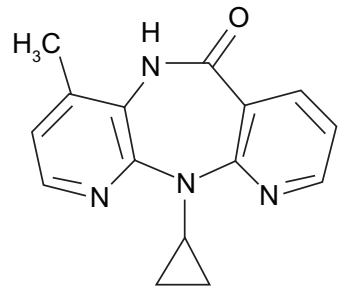

Nevirapine

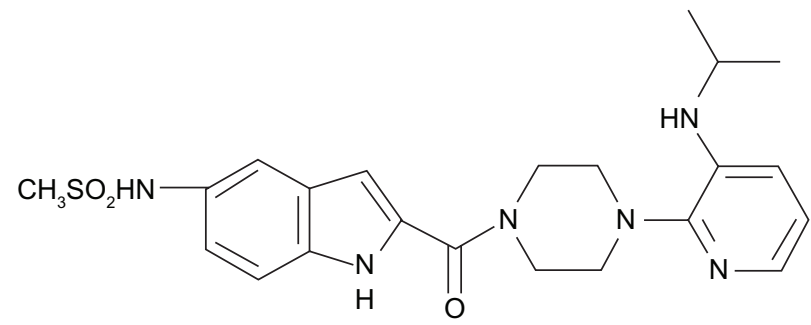

\section{Delavirdine}

Figure 2 Chemical structures of first-generation (efavirenz, nevirapine, and delavirdine) and second-generation (etravirine) nonnucleoside reverse transcriptase inhibitors. 


\section{Pharmacokinetics}

The pharmacokinetics of etravirine are based on studies utilizing 3 oral formulations: the polyethylene glycol (PEG) 4000-based $50 \mathrm{mg}$ capsule (TF002) as etravirine base used in early phase I and phase IIa studies, the hydroxypropylmethylcellulose (HPMC) $200 \mathrm{mg}$ tablet formulation (TF035) as etravirine hydrogen bromide salt used in late phase I and phase II studies, and the spray-dried HPMC $100 \mathrm{mg}$ tablet formulation (F060) as amorphous etravirine base as the current commercial product used in phase III trials. Formulation modifications were made to improve bioavailability due to the poor solubility exhibited by etravirine and to reduce tablet size. ${ }^{13}$ A $25 \mathrm{mg}$ spray-dried HPMC tablet is currently being investigated for children and adolescents. ${ }^{14}$

Etravirine is slowly absorbed, with peak plasma concentrations achieved after approximately 4 hours (range, 2-8 hours) with the current commercial formulation. ${ }^{15}$ Although the oral bioavailability of etravirine has not been established, administration with food increases apparent bioavailability by up to $50 \%$ and decreases intersubject variability. Significant differences in absorption are not evident with varying food compositions. ${ }^{16}$ Two hundred milligrams of the F060 amorphous tablet formulation used commercially achieves 30\%-70\% higher systemic exposure compared with $800 \mathrm{mg}$ of the TF035 hydrogen bromide salt used in phase II studies. ${ }^{13}$ Use of acid suppressants (eg, antacids, $\mathrm{H} 2$ receptor antagonists, and proton pump antagonists) does not alter etravirine absorption; although coadministration with omeprazole does increase etravirine systemic exposure, presumably due to impaired CYP2C19 metabolism. ${ }^{17}$ Dispersion of F060 tablets in a glass of water prior to ingestion does not result in reduced bioavailability and represents an alternative oral administration option. ${ }^{14}$ Serum concentrations decline with repeated dosing due to autoinduction of metabolism. ${ }^{13}$ The elimination half-life for etravirine is approximately 30-40 hours. Interpatient and intrapatient variability in pharmacokinetics in HIV-infected patients are $60 \%$ and $40 \%$, respectively. ${ }^{15}$ Higher etravirine systemic exposure is noted with decreased body weight and hepatitis $\mathrm{B}$ or $\mathrm{C}$ coinfection (1.35-fold) but does not warrant dosage modification. ${ }^{18}$ Serum concentrations achieved in patients with mild-to-moderate hepatic impairment (Child-Pugh class A or class B) are similar to healthy subjects and do not require dosage adjustment. ${ }^{19}$ In a single case of a woman with decompensated liver disease, etravirine serum concentrations were 60 -fold higher than projected on standard dosing, with an estimated half-life of 237 hours, indicating that etravirine should be avoided in patients with severe hepatic impairment (Child-Pugh class C). ${ }^{20}$ Etravirine is $99.9 \%$ bound to plasma protein, primarily to albumin and alpha ${ }_{1}$-acid glycoprotein, in a concentration-independent manner. ${ }^{14}$ Modest reduction $(29 \%)$ in etravirine serum concentration was observed in a single patient receiving hemodialysis 3 times weekly on standard etravirine doses, signifying that dosage modification with dialysis is not likely necessary. ${ }^{21}$ The extent of etravirine distribution into the central nervous system and other tissues has not been fully characterized. ${ }^{13,14}$ A summary of etravirine pharmacokinetics is given in Table 1.

\section{Metabolism and drug interactions}

Etravirine is metabolized by cytochrome P450 (CYP) 3A4, CYP2C9, and CYP2C19 and has the potential for drug interactions with coadministered medications. It exhibits a mixed interaction profile, as illustrated by its potential to induce CYP3A4, CYP2B6, and uridine diphosphateglucuronosyltransferase and inhibits CYP2C9 (inhibition constant $\left[K_{\mathrm{i}}\right]=0.25 \mu \mathrm{g} / \mathrm{mL}$ ) and CYP2C19. ${ }^{13}$ Etravirine is also an inhibitor of p-glycoprotein, with an apparent $50 \%$ inhibition concentration $\left(\mathrm{IC}_{50}\right)$ of $24.2 \mu \mathrm{M}$. Low amounts of etravirine (1.2\%) are eliminated in urine; over $80 \%$ of an administered dose is excreted unchanged in the feces. ${ }^{13}$

Drug interactions between etravirine and some antiretrovirals have been described. Etravirine decreases unboosted atazanavir, indinavir, and saquinavir and should not be used with these agents in the absence of ritonavir. ${ }^{14}$ Etravirine also decreases atazanavir boosted with low-dose ritonavir, but the clinical significance of this is unknown. ${ }^{22}$ Amprenavir systemic exposure is increased with etravirine; therefore, caution should be exercised in using combined etravirine and fosamprenavir/ritonavir therapy. ${ }^{23}$ Tipranavir/ritonavir significantly lowers etravirine serum concentrations (70\%$80 \%$ ) and should not be used concurrently. ${ }^{24}$ Etravirine can be safely used in combination with lopinavir/ritonavir,

Table I Summary of etravirine pharmacokinetics ${ }^{\mathrm{a}, 13,15,18}$

\begin{tabular}{ll}
\hline Tmax, median (range) & $4 \mathrm{~h}(2-8)$ \\
Half-life, mean & $30-40 \mathrm{~h}$ \\
Vd & $422 \mathrm{~L}$ \\
Cmax, mean $( \pm S D)$ & $45 \mathrm{I} \mathrm{ng} / \mathrm{mL}( \pm 232)$ \\
Cmin, mean $( \pm S D)$ & $185 \mathrm{ng} / \mathrm{mL}( \pm 128)$ \\
AUC $_{0-12 \mathrm{~h}}$, mean $( \pm S D)$ & $3,713 \mathrm{ng} \cdot \mathrm{h} / \mathrm{mL}( \pm 2,069)$ \\
Protein binding & $99.8 \%-99.9 \%($ concentration-independent) \\
\hline
\end{tabular}

Note: aBased on F060 commercial formulation.

Abbreviation: SD, standard deviation. 
ritonavir-boosted darunavir, and saquinavir. ${ }^{14}$ Concurrent use of etravirine with other NNRTIs has not been shown to be beneficial and is not recommended; reduction in etravirine serum concentrations is anticipated if combined with either efavirenz or nevirapine. ${ }^{13,25}$ No clinically significant interactions between etravirine and tenofovir and didanosine have been identified ${ }^{26,27}$ Interactions with other nucleoside reverse transcriptase inhibitors (NRTIs) are not expected to occur. ${ }^{13}$ Etravirine reduces serum concentrations of the CCR5 receptor antagonist, maraviroc, and requires different dosage adjustments based on whether a boosted protease inhibitor is included in the regimen. ${ }^{28}$ Minimum concentrations of the integrase inhibitor, raltegravir, are lower with concurrent etravirine in healthy volunteers and were associated with decreased virologic response in $4 \mathrm{HIV}$-infected patients; however, the clinical relevance of this is uncertain. ${ }^{29-31}$

Interactions between etravirine and medications for other comorbidities have also been described. Etravirine decreases the concentrations of clarithromycin, atorvastatin, and sildenafil and may require the use of higher doses of these agents or selection of alternative therapy. ${ }^{32-34}$ The use of etravirine with other statins (eg, simvastatin and lovastatin) and phosphodiesterase-5 inhibitors (taladafil and vardenafil) has not been studied but may require dosage adjustments with some agents due to similar metabolism through CYP3A4. ${ }^{35}$
Digoxin serum concentrations are increased by etravirine, potentially due to the inhibition of p-glycoprotein, but are not thought to be clinically significant. ${ }^{36}$ A $35 \%$ reduction in etravirine area under the curve (AUC) occurs with rifabutin but is not thought to be clinically significant unless a boosted protease inhibitor is also coadministered. ${ }^{37}$ Administration of etravirine with potent enzyme inducers (eg, rifampin, phenytoin, phenobarbital, and carbamazepine) is not recommended due to the potential for subtherapeutic etravirine concentrations. ${ }^{35}$ In studies conducted in healthy volunteers, etravirine increased single-dose warfarin $10 \mathrm{mg}$ (S-warfarin AUC ratio with and without etravirine $=1.05 ; 90 \%$ confidence interval, 0.93-1.19), indicating that etravirine has the potential to alter warfarin metabolism by inhibition of CYP2C9. ${ }^{38}$ The labeling of etravirine was recently updated to include information regarding interactions with fluconazole, voriconazole, and clopidogrel. ${ }^{35}$ Coadministration of fluconazole or voriconazole with etravirine significantly increased the etravirine concentrations. The clinical implications of this increase are unknown and, as such, these combinations should be used with caution. Inhibition of $2 \mathrm{C} 19$ by etravirine may decrease the concentration of the active metabolite of clopidogrel and, therefore, decrease its therapeutic effect. Use of an alternate agent is suggested. A summary of the effect of etravirine on coadministered drugs is given in Table 2.

Table 2 The Effect of etravirine on the pharmacokinetics of coadministered drugs ${ }^{13,14}$

\begin{tabular}{|c|c|c|c|c|}
\hline \multirow[t]{2}{*}{ Affected drug (dose/schedule) ${ }^{a}$} & \multirow[t]{2}{*}{ 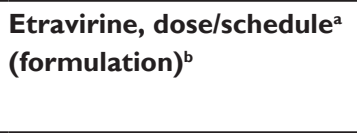 } & \multicolumn{3}{|c|}{$\begin{array}{l}\text { Mean ratio of affected drug } \\
\text { with/without etravirine }\end{array}$} \\
\hline & & AUC & $C_{\max }$ & $C_{\min }$ \\
\hline \multicolumn{5}{|l|}{ Antiretrovirals } \\
\hline Atazanavir (400 mg QD) & 800 mg BID (TF035) & 0.83 & 0.97 & 0.53 \\
\hline Atazanavir/ritonavir (300/100 mg QD) & 800 mg BID (TF035) & 0.86 & 0.97 & 0.62 \\
\hline Darunavir/ritonavir (600/l00 mg BID) & 200 mg BID (F060) & 1.15 & I.II & 1.02 \\
\hline Fosamprenavir/ritonavir (700//00 mg BID) & 800 mg BID (TF035) & 1.69 & 1.62 & 1.77 \\
\hline Indinavir (800 mg TID) & I,600 mg BID (TF035) & 0.54 & 0.72 & 0.24 \\
\hline Lopinavir/ritonavir (400/I00 mg BID) & 800 mg BID (TF035) & 0.82 & 0.84 & 0.76 \\
\hline Maraviroc (300 mg BID) & 200 mg BID (F060) & 0.47 & 0.40 & 0.61 \\
\hline Maraviroc (I50 mg BID) & 200 mg BID (F060) & 3.10 & 1.77 & 5.27 \\
\hline Raltegravir (400 mg BID) & 200 mg BID (F060) & 0.90 & 0.89 & 0.66 \\
\hline Saquinavir $(\mathrm{I}, 200 \mathrm{mg} \times \mathrm{I})$ & 900 mg BID (TF002) & 0.54 & 0.48 & NR \\
\hline Saquinavir/ritonavir (I,000/I00 mg BID) & 800 mg BID (TF035) & 0.95 & 1.00 & 0.80 \\
\hline \multicolumn{5}{|l|}{ Other medications } \\
\hline Atorvastatin (40 mg QD) & 800 mg BID (TF035) & 0.63 & 1.04 & NR \\
\hline Clarithromycin (500 mg BID) & 200 mg BID (F060) & 0.61 & 0.66 & 0.47 \\
\hline Digoxin $(0.5 \mathrm{mg} \times \mathrm{I})$ & 200 mg BID (F060) & 1.18 & 1.19 & NR \\
\hline Sildenafil $(50 \mathrm{mg} \times \mathrm{I})$ & 800 mg BID (TF035) & 0.43 & 0.55 & NR \\
\hline
\end{tabular}

Notes: aAll evaluations were conducted under steady-state conditions unless otherwise noted; ${ }^{\mathrm{b}} \mathrm{TF035}=\mathrm{HPMC}$ etravirine hydrogen bromide tablet, F060 = spray-dried HPMC amorphous etravirine base tablet, TF002 = PEG 4000 etravirine base capsule. 'Administered in combination with darunavir/ritonavir (600/I00 mg BID). Adapted from European Medicines Agency. ${ }^{13}$

Abbreviations: QD, once daily; BID, twice daily; TID, thrice daily; NR, not reported. 


\section{Resistance}

Etravirine exhibits a higher genetic barrier to resistance than other NNRTIs because 3 or more resistance-associated mutations (RAMs) are typically necessary to cause clinical resistance vs a single mutation with first-generation NNRTIs. ${ }^{39-41}$ In addition, presence of the K103N (lysine to arginine) mutation that commonly confers resistance to first-generation NNRTIs is not associated with decreased virologic response to etravirine. ${ }^{42}$ Seventeen RAMs have been associated with decreased susceptibility to etravirine. The magnitude of resistance is determined by the number and the weighted genotype mutation score for individual mutations; an example of the DUET weighted score is outlined in Table $3 .{ }^{42}$ Some discordance in etravirine susceptibility interpretation currently exists between different genotype algorithms and may be related to variations in mutation selection criteria and weight assignment for individual mutations (eg, Y181C and L100I) ${ }^{43}$ Summation of weighted mutation scores has been correlated with clinical response, where DUET weighted scores of 0-2 were associated with a $74 \%$ virologic response rate, whereas scores of $2.5-3.5$ and $\geq 4.0$ were associated with viral response rates of $52 \%$ and $38 \%$, respectively. ${ }^{42}$ The highest change in $\mathrm{EC}_{50}$ occurs with the combination of V179F with either Y181C or Y181I. ${ }^{13}$ The prevalence of baseline clinical resistance to etravirine among patients leading to NNRTI treatment failure ranges from $4.6 \%$ to $13 \% .{ }^{44}{ }^{47}$ Failure of nevirapine therapy is associated with a greater likelihood for the development of etravirine RAMs compared with the failure of efavirenz ${ }^{44,46-48}$ Patients with less than 3-fold change in $\mathrm{EC}_{50}$ are more likely to achieve viral suppression with etravirine than patients above this threshold, which is used as the lower cutoff for phenotypic resistance assays; $\mathrm{a} \geq 13$-fold reduction in phenotypic susceptibility represents the upper clinical cutoff and is associated with the absence of clinical response to etravirine. ${ }^{42}$

\section{Clinical efficacy}

The clinical efficacy of etravirine in adult HIV-infected patients has been established based on results from

Table 3 Etravirine resistance-associated mutations (RAMS)

\begin{tabular}{ll}
\hline Mutations & Genotypic score $^{\text {a }}$ \\
\hline YI8II, YI8IV & 3.0 \\
LI00I, KI0IP, YI8IC, M230L & 2.5 \\
VI06I, EI38A, VI79F, GI90S & 1.5 \\
V90I, A98G, KI0IE, KI0IH, VI79D, & 1.0 \\
VI79T, GI90A & \\
\hline
\end{tabular}

Note: ${ }^{a}$ The relative weight assigned to each individual mutation signifying the magnitude of change in susceptibility using the DUET weighted score. ${ }^{42}$ Cumulative scores greater than 4 are associated with the lowest virologic response to etravirine. phase II and phase III studies. These studies examined the use of etravirine in antiretroviral treatment-experienced patients and in treatment-naive subjects. The efficacy of etravirine in special populations (pregnant women and pediatric patients) in well-controlled studies is currently being investigated. ${ }^{49}$

\section{Proof of principle studies (phase lla)}

TMC125-C208 was a randomized, double-blind, placebocontrolled trial to assess the short-term antiviral activity, safety, tolerability, and pharmacokinetics of etravirine monotherapy in treatment-naive and HIV-infected individuals. Nineteen subjects were randomized 2:1 to receive etravirine $900 \mathrm{mg}$ as PEG 4000 capsule formulation or placebo. Patients received medication twice daily for 7 days within 15 minutes of a meal. The primary efficacy parameter was the change in plasma HIV-1 RNA from baseline. Tolerability and safety were evaluated using patient-reported adverse effects and laboratory changes. After 7 days of treatment, a mean decrease in plasma HIV-1 RNA of $1.99 \log _{10}$ copies/mL was observed (range, 3.39-1.13) in patients receiving etravirine compared with $0.06 \log _{10}$ copies $/ \mathrm{mL}$ in the placebo group. Viral decay was calculated at a rate of $0.33 \log _{10}$ copies $/ \mathrm{mL}$ per day in the treatment group compared with $0.02 \log _{10}$ copies/mL per day in the placebo group $(P<0.001)$. All subjects in the etravirine treatment arm achieved $\geq 1 \log _{10}$ reduction in viral load; 8 subjects achieved a plasma HIV-1 RNA $<400$ copies $/ \mathrm{mL}(P<0.001)$ and 2 were $<50$ copies $/ \mathrm{mL}(P=\mathrm{NS})$. Patients receiving etravirine also had a significant increase in CD4 cells compared with the placebo group $(+104$ and -150 cells $/ \mu \mathrm{L}$, respectively; $P=0.016) .^{50}$

TMC 125-C207 was an open-label study on the efficacy, tolerability, safety, and pharmacokinetics of etravirine monotherapy in HIV-1-infected subjects who fail a current NNRTI-containing regimen. Sixteen patients on an NNRTIcontaining regimen (efavirenz or nevirapine) with plasma HIV-1 RNA > 2,000 copies/mL and confirmed phenotypic resistance to efavirenz were included in the study. Patients were given etravirine $900 \mathrm{mg}$ PEG 4000 capsule twice daily for 7 days. Antiretroviral therapy selected on the basis of resistance testing obtained during study screening was initiated on day 8 . The primary outcome measure was the mean plasma HIV-1 RNA decay rate over the 7-day treatment period. Fifteen patients completed the study. A plasma HIV-1 RNA decay rate of $0.13 \log _{10}$ copies $/ \mathrm{mL}$ per day was observed during the treatment period $(P<0.001)$. Viral load decrease ranged from 0.18 to $1.71 \log _{10}$ copies $/ \mathrm{mL}$ with a median decrease of $0.89 \log _{10}$ copies $/ \mathrm{mL}$. Seven (44\%) patients had a decrease in viral load of at least $1.0 \log _{10}$ and $12(75 \%)$ had a decrease 
of at least $0.5 \log _{10}$. Two patients achieved plasma HIV-1 RNA $<400$ copies $/ \mathrm{mL}$. There were no significant changes in either mean CD4 count or CD4 percentage. The extent of resistance to efavirenz and etravirine at baseline was 116.1-fold and 2.2-fold, respectively. At the end of therapy, no new NNRTIassociated resistance mutations were identified. ${ }^{51}$

\section{Dose-ranging studies (phase Ilb)}

TMC 125-C203 was a 48-week, double-blind, placebocontrolled, 2-stage dose escalation study conducted in HIV1-infected subjects with prior NRTI, protease inhibitor, and NNRTI experience. Patients were randomly assigned to the 400, 800, and 1,200 mg TF035 tablet formulations of etravirine administered twice daily or to placebo, in combination with optimized background therapy (OBT). Patients enrolled in stage 1 were randomized in a 1:1:1 fashion to receive etravirine $400 \mathrm{mg}$, etravirine $800 \mathrm{mg}$, or placebo twice daily. Patients enrolled in stage 2 were randomized in a 1:2:4 manner to receive placebo, etravirine $800 \mathrm{mg}$, or etravirine 1,200 mg administered twice daily. The primary end point was safety over 48 weeks; plasma HIV-1 RNA measurements were collected and reported but could not be reliably interpreted due to the modification of viral load collection technique during the study. A total of 240 patients were enrolled in the trial (166 patients in stage 1 and 74 patients in stage 2), with 57, 74, 43, and 66 subjects enrolled in the $400,800,1,200 \mathrm{mg}$, and placebo arms, respectively, for the initial 48-week period. ${ }^{13}$ Patients were allowed to extend treatment beyond 48 weeks. More patients in the etravirine group received de novo enfuvirtide as OBT. Viral load results were reported only for samples collected using EDTA tubes. No significant difference in viral load reduction was noted between the combined etravirine groups and placebo group during stage 1 at weeks 24 and 48; however, a significant difference was observed at week 24 in the $800 \mathrm{mg}$ and $1,200 \mathrm{mg}$ etravirine groups compared with placebo $(P=0.009$ and $P=0.049$, respectively). Similarly, no significant differences in CD4 change were noted between groups in stage 1 . Significant differences between the $800 \mathrm{mg}$ and $1,200 \mathrm{mg}$ etravirine groups and the placebo group were observed during stage $2(P=0.025$ and $P=0.019$, respectively). CD4 changes were also greater in the 2 etravirine groups $(P=0.025$ and $P=0.019$ for the $800 \mathrm{mg}$ and $1,200 \mathrm{mg}$ groups, respectively, compared with placebo). No difference in safety was noted between the etravirine groups and the placebo group..$^{52}$

TMC 125-C223 was a 48-week, partially blinded, controlled, multicenter, dose-ranging trial conducted in 199 treatment-experienced HIV-1-infected subjects with baseline
NNRTI resistance and 3 or more primary protease inhibitor mutations. Patients were randomized in a 1:2:2 fashion to the active control group or etravirine $400 \mathrm{mg}$ or $800 \mathrm{mg}$ TF035 tablet formulation given twice daily. Investigators and study subjects were blinded to etravirine dose but not to assignment to etravirine therapy or control. Antiretroviral therapy in the control arm consisted of at least 3 approved antiretrovirals that included NRTIs, protease inhibitors, and enfuvirtide or a combination of all 3. Patients assigned to the 2 etravirine groups also received investigator-selected OBT consisting of at least 2 approved antiretrovirals that included NRTIs, lopinavir/ritonavir or enfuvirtide, or a combination of all 3. The primary end point was the mean change in plasma HIV-1 RNA from baseline. A total of 199 patients $(n=80$ etravirine $400 \mathrm{mg}, \mathrm{n}=79$ etravirine $800 \mathrm{mg}, \mathrm{n}=40$ placebo) were randomized and included in the intent-to-treat analysis. At 48 weeks, the mean change in plasma HIV-1 RNA was $-0.88,-1.01$, and $-0.14 \log _{10}$ copies $/ \mathrm{mL}$ in the etravirine $400,800 \mathrm{mg}$, and control groups, respectively $(P=0.018$ and $P=0.002$ for the $400 \mathrm{mg}$ and $800 \mathrm{mg}$ groups, respectively, compared with control). The proportion of patients achieving plasma HIV-1 RNA $<50$ copies $/ \mathrm{mL}$ was $22.5 \%$ and $21.5 \%$ in the $400 \mathrm{mg}$ and $800 \mathrm{mg}$ etravirine groups, respectively, vs none in the control group $(P<0.001)$. From subgroup analysis, greater reduction in viral load was achieved in patients receiving etravirine $800 \mathrm{mg}$ vs $400 \mathrm{mg}$ twice daily when either enfuvirtide or a protease inhibitor were not included in the regimen. Greater response with $800 \mathrm{mg}$ etravirine twice daily was also observed with increased number of active agents in the regimen. The number of etravirine RAMs at baseline was predictive of response to etravirine, with the greatest reductions in viral load occurring in patients with the fewest mutations. CD4 cell count increases appeared to be greater in the etravirine groups compared with the control group but did not achieve statistical significance. A large number of patients $(\mathrm{n}=100)$ discontinued the study prematurely due to virologic failure, with a greatest proportion occurring in the control group (97.5\%) compared with the combined etravirine arms (38.4\%). Given the greater virologic response with the $800 \mathrm{mg}$ vs $400 \mathrm{mg}$ etravirine dose in the absence of concomitant enfuvirtide use and no apparent differences in safety, the $800 \mathrm{mg}$ dose was selected for further phase III studies. ${ }^{53,54}$

TMC125-C227 was a randomized, controlled, open-label, exploratory study in protease inhibitor-naive patients with documented NNRTI resistance. Eligibility criteria included plasma HIV-1 RNA $\geq 1,000$ copies/mL. Women who had developed NNRTI resistance as a result of receiving 
previous NNRTI treatment to prevent mother to child HIV transmission were allowed to participate and comprised $25 \%$ of the study population. Patients with chronic hepatitis $\mathrm{B}$ or $\mathrm{C}$ infection could participate if liver transaminases were less than 3 times the upper limit of normal. Patients were randomly assigned (1:1) to etravirine TF035 formulation $800 \mathrm{mg}$ twice daily or to an investigator-selected protease inhibitor, both in combination with 2 NRTIs selected by the investigator. A total of 116 patients $(n=59$ etravirine, $\mathrm{n}=57$ control) were included in the intent-to-treat population. Ninety-six percent of patients in the control group received a ritonavir-boosted protease inhibitor. An unplanned interim analysis was conducted at week 12 and showed a change in mean plasma HIV-1 RNA of $-1.39 \log _{10}$ in the etravirine group vs $-2.16 \log _{10}$ in the control group, indicating that virologic response in the etravirine arm was inferior. CD4 response was similarly lower in the etravirine group compared with controls at week 12 (42 and 79 cells $/ \mathrm{mm}^{3}$, respectively). Enrollment in the etravirine arm was subsequently discontinued, and patients originally assigned to etravirine treatment were switched to investigator-selected protease inhibitor therapy for the remaining duration of follow-up. Post hoc analysis of treatment response based on baseline resistance showed that a greater proportion of patients in the etravirine group had preexisting NRTI resistance, including thymidine analog mutations, M184I/V, and K65R. Thirtyone percent of etravirine patients had no active NRTIs in their antiretroviral regimen compared with $23 \%$ of control patients. Although the presence of any NNRTI RAM at baseline was also associated with reduced virologic response, the number of mutations did not predict response unless the mutations were etravirine RAMs. Presence of 3 or more etravirine RAMs was associated with the greatest reduction in virologic response, especially when Y181C was present. Patient who showed increased virologic response were more likely to have an etravirine phenotypic fold-change less than $4\left(-1.82 \log _{10} ; \mathrm{n}=24\right)$ at baseline compared with those with a fold change greater than $4\left(-0.96 \log _{10} ; n=11\right)$. Although viral load reductions were numerically greater in patients with etravirine $\mathrm{AUC}_{12}$ exceeding 4,521 $\mathrm{ng} \cdot \mathrm{h} / \mathrm{mL}$, no significant relationship between viral load response and etravirine systemic exposure was noted. ${ }^{55}$

The ANRS 139 TRIO study was a multicenter, uncontrolled trial assessing the efficacy and safety of antiretroviral regimens containing etravirine, darunavir/ritonavir, and raltegravir in antiretroviral treatment-experienced patients in 49 clinics in France. Eligibility criteria included plasma HIV-1 RNA $>1,000$ copies $/ \mathrm{mL}, \geq 3$ protease inhibitor mutations, and $\geq 3$ NRTI mutations. Patients with a history of virologic failure during treatment with a first-generation NNRTI were also eligible provided in vitro susceptibility to etravirine was evident at screening. Patients with chronic hepatitis B or C infection with Child-Pugh scores of B or $\mathrm{C}$ without cirrhosis or liver function tests less than 3 times the upper limit of normal were also included. Etravirine $200 \mathrm{mg}$ twice daily using the F060 tablet formulation was administered with raltegravir $400 \mathrm{mg}$ twice daily and darunavir/ritonavir 600/100 mg twice daily. OBT consisted of investigator-selected NRTIs with or without enfuvirtide. The primary end point was the proportion of patients with plasma HIV-1 RNA < 50 copies/mL at week 24; secondary end point included the proportion of patients with plasma HIV-1 RNA $<50$ copies/mL at week 48. A total of 103 patients were enrolled in the study and comprised the intent-to-treat population. One hundred patients (97\%) remained on therapy at week 24 . The median duration of prior antiretroviral therapy for the study population was 13 years. Viral suppression to $<50$ copies/mL was observed in $90 \%$ of patients at week 24 and $86 \%$ of patients at week 48 . Five of the 10 patients with plasma HIV-1 RNA $>50$ copies $/ \mathrm{mL}$ had viral loads $<400$ copies/mL. Eighty-four percent of patients in the study received NRTIs as their OBT; $12 \%$ had enfuvirtide included in their OBT. The median changes in viral load and CD4 at week 48 were $-2.4 \log _{10}$ and +108 cells/ $\mathrm{mm}^{3}$, respectively. ${ }^{56}$

\section{Large safety and efficacy studies (phase III)}

The efficacy and safety of etravirine in antiretroviral treatment-experienced patients with preexisting NNRTI resistance have been established on the basis of 2 large multicenter phase III clinical trials (DUET 1 and DUET 2) with identical study designs.

TMC125-C206 (DUET 1) was a randomized, doubleblind, placebo-controlled trial conducted in South America, Mexico, Puerto Rico, Panama, Thailand, France, and the United States. Inclusion criteria included a plasma HIV-1 RNA $\geq 5,000$ copies $/ \mathrm{mL}$, presence of $\geq 3$ primary protease inhibitor mutations, and at least 1 NNRTI RAM. Patients with chronic hepatitis $\mathrm{B}$ and $\mathrm{C}$ coinfection were permitted to enroll, provided liver transaminases were within 5 times the upper limit of normal and treatment was not thought to be necessary by the investigator. Study subjects were randomly assigned (1:1) to etravirine $200 \mathrm{mg}$ using the $100 \mathrm{mg}$ F060 tablet formulation or placebo twice daily in combination with low-dose ritonavir-boosted darunavir 
and investigator-selected NRTIs. Use of enfuvirtide was optional. Randomization was stratified according to use or reuse of enfuvirtide, prior darunavir use, and baseline plasma HIV-1 RNA $\geq 30,000$ copies/mL. The primary end point was the proportion of patients with plasma HIV-1 RNA $<50$ copies/mL at 24 weeks. A total of 612 patients ( $n=304$ etravirine, $n=308$ placebo) were included in the intent-to-treat population. After 24 weeks of therapy, 56\% and $39 \%$ of patients in the etravirine and placebo groups, respectively, achieved plasma HIV-1 RNA $<50$ copies/mL $(P=0.005)$. Plasma HIV-1 RNA decreased from baseline by a mean of $2.41 \log _{10}$ copies/mL compared with $1.70 \log _{10}$ copies $/ \mathrm{mL}$ in the 2 treatment arms, respectively $(P<0.0001)$. CD4 cell counts increased from baseline by 89 cells $/ \mathrm{mm}^{3}$ in the etravirine arm and 64 cells $/ \mathrm{mm}^{3}$ in the placebo arm $(P=0.0002)$. Patients who reused or did not use enfuvirtide were more likely to achieve a plasma HIV-1 RNA $<50$ copies $/ \mathrm{mL}$ in the etravirine arm than in the placebo arm (55\% and 33\%, respectively; $P<0.0001$ for the difference). However, no significant difference in the proportion of patients achieving the primary end point was observed between the groups with de novo enfuvirtide use. ${ }^{41}$

TMC125-C216 (DUET 2) was a randomized, doubleblind, placebo-controlled trial conducted in Australia, Canada, Europe, Poland, Portugal, and the United States. Inclusion criteria, assignment to therapy, and prespecified analyses were identical to DUET 1. A total of 591 patients ( $\mathrm{n}=295$ etravirine, $\mathrm{n}=296$ placebo) comprised the intent-to-treat population. After 24 weeks of therapy, $62 \%$ and $44 \%$ of patients in the etravirine and placebo groups, respectively, achieved plasma HIV-1 RNA $<50$ copies $/ \mathrm{mL}$ $(P=0.0003)$. Plasma HIV-1 RNA decreased from baseline by $2.34 \log _{10}$ copies $/ \mathrm{mL}$ compared with $1.68 \log _{10}$ copies $/ \mathrm{mL}$ in the 2 treatment arms, respectively $(P<0.0001)$. CD4 cell counts increases between the 2 groups were not significantly different. Greater virologic suppression was observed in the etravirine group than in the placebo group, regardless of the number of active drugs in the accompanying antiretroviral regimen. However, no significant difference in the proportion of patients with plasma HIV-1 RNA $<50$ copies/mL or $<400$ copies $/ \mathrm{mL}$ was present when de novo enfuvirtide was controlled for in the 2 study groups. The 2 groups were well balanced regarding enfurvirtide use, with $27 \%$ receiving enfuvirtide de novo and $25 \%$ reusing it in both groups. ${ }^{40}$

Virologic response to etravirine was sustained beyond the initial 24 weeks of therapy. In the pooled DUET-1 and DUET-2 preplanned analysis, $61 \%$ of patients in the combined etravirine group had plasma HIV-1 RNA $<50$ copies/mL compared with $40 \%$ of patients in the combined placebo group at week 48. Plasma HIV-1 RNA decreased by $2.25 \log _{10}$ copies/mL and $1.49 \log _{10}$ copies $/ \mathrm{mL}$ in the etravirine and placebo groups, respectively $(P<0.0001)$. CD4 cell counts increased from baseline by 98 cells $/ \mathrm{mm}^{3}$ vs 73 cells $/ \mathrm{mm}^{3}$ in the etravirine and placebo groups, respectively $(P=0.0006)$. By week 48 , the pooled etravirine group vs the placebo group achieved higher virologic responses regardless of the number of active agents used in the background regimen. ${ }^{37,38}$ At 96 weeks, $57 \%$ vs $36 \%$ had plasma HIV-1 RNA $<50$ copies/mL in the etravirine and placebo groups, respectively $(P<0.0001)$, demonstrating long-term efficacy with etravirine. ${ }^{57}$

\section{Adverse events}

The most common adverse events associated with etravirine occurring at a higher rate than placebo are the development of cutaneous reactions (19\%). Cutaneous reactions typically manifest as a maculopapular rash after a median of 14 days and are mild to moderate in severity. In phase III studies, $1.3 \%$ of patients developed grade 3 or 4 rashes while receiving etravirine vs $0.2 \%$ receiving placebo. Overall, $2.2 \%$ of patients in phase III trials discontinued therapy because of rash. The majority of rashes occurred within the first 4 weeks of therapy. Resolution is reported to occur within 1-2 weeks (median, 15 days) on continued therapy. Women appear to be at greater risk than men (30\% vs $18 \%$ frequency of rash in women and men in the DUET studies, respectively); however, the severity of rash does not appear to differ. History of a rash with other NNRTIs is not predictive of the risk for cutaneous reactions with etravirine. ${ }^{58}$ In postmarketing reports, rare cases $(<0.1 \%)$ of severe, life-threatening skin reactions (StevenJohnson syndrome, toxic epidermal necrolysis, and erythema multiforme) have prompted revised safety labeling. ${ }^{35}$ These reactions have included rash, constitutional symptoms, and occasionally include organ dysfunction (eg, hepatic failure). Gastrointestinal effects are primarily nausea (14\%), diarrhea (19\%), and abdominal pain that are mild in severity. No significant changes in adverse event profile were notable in patients who continued etravirine therapy to 48 or 96 weeks. ${ }^{58,59}$

Etravirine does not exhibit significant potential to alter cardiac conduction. A randomized, double-blind, placebo/activecontrolled study in $41 \mathrm{HIV}$-seronegative volunteers showed no prolongation of QTc interval. ${ }^{60}$ Etravirine has also not been demonstrated to cause birth defects in animal studies and is labeled as pregnancy category B. However, because safety has not been established in infants, women should not breastfeed while receiving etravirine. ${ }^{35}$ Limited data are available regarding the safety of etravirine during pregnancy. Etravirine was 
shown to be safe and effective when combined with darunavir/ ritonavir, enfuvirtide, and tenofovir/emtricitabine at 25 weeks gestation in a pregnant HIV-infected woman with multiclass resistance. Healthy twin babies were delivered at 34 weeks gestation and remained seronegative after 4 months. Cord blood collected at delivery revealed detectable levels of etravirine. ${ }^{61}$ The safety and pharmacokinetics of etravirine were examined in 5 women who received etravirine as part of compassionate use programs. Three women began etravirine with other antiretroviral therapy during the first trimester and the other 2 in the third trimester. With the exception of one preterm delivery of twin babies by caesarian section, the remaining 4 women delivered at term ( 2 caesarian and 2 vaginal). One baby was born with a minor ear malformation, but all children were born healthy and remained HIV seronegative. Etravirine pharmacokinetics during pregnancy were consistent with nonpregnant adults. ${ }^{62}$ Use of etravirine and darunavir/ritonavir in the second and third trimesters, respectively, is being examined in an ongoing pharmacokinetic study. ${ }^{49}$

\section{Health-related quality of life}

The DUET-1 and DUET-2 trials included an examination of health-related quality of life as measured by the Functional Assessment of HIV Infection questionnaire. Baseline and 24 -week results of the questionnaire were compared. The 47 items in the questionnaire are grouped into 5 subscales, but only the Physical Well-Being and Functional and Global Well-Being scales were analyzed. A total of 1,203 patients were included in the analysis $(n=599$ etravirine, $n=604$ placebo). Statistically significant improvement in both Physical Well-Being and Functional and Global Well-Being scales were seen in the etravirine-treated group at week 24 compared with baseline ( $P<0.0001$ and $P=0.0003$, respectively). Compared with the placebo group, these differences remained statistically greater for both measures $(P=0.01$ for Physical Well-Being; $P=0.03$ for Functional and Global Well-Being). ${ }^{63}$

\section{Dosage and administration}

Etravirine is presently approved for combination therapy with other antiretroviral agents in adult HIV-infected individuals with documented or suspected resistance to first-generation NNRTIs. It is available as the $100 \mathrm{mg}$ F060 tablet in the recommended dose of $200 \mathrm{mg}$ twice daily following a meal. The tablets can also be dispersed in a glass of water if patients have difficulty swallowing the tablet. The glass should be rinsed several times to assure the entire dose is achieved. It should be noted that unlike the NNRTI, efavirenz, the activity of etravirine in combination with 2 NRTIs for treatment-naive individuals is not presently established. ${ }^{55,64}$

\section{Conclusion}

Etravirine is the first second-generation NNRTI and possesses potent activity against HIV-1 strains that display resistance to first-generation NNRTI agents. The most common adverse effect is the development of rash. Etravirine is currently indicated only for use in combination therapy for antiretroviral treatment-experienced patients with documented or suspected resistance to first-generation NNRTIs. Drug interactions with some antiretrovirals can preclude use of etravirine in some antiretroviral regimens.

\section{Disclosure}

The authors disclose no conflicts of interest.

\section{References}

1. UNAIDS. AIDS epidemic update. 2009 Dec.

2. Hall HI, Ruiguang S, Rhodes P, et al. Estimation of HIV incidence in the United States. JAMA. 2008;300:520-529.

3. Pallela FJ, Delaney KM, Moorman AC, et al. Declining morbidity and mortality among patients with advanced human immunodeficiency virus infection. N Engl J Med. 1998;338:853-860.

4. Panel on Antiretroviral Guidelines for Adults and Adolescents. Guidelines for the use of antiretroviral agents in HIV-1-infected adults and adolescents. Department of Health and Human Services. 2009 Dec;1-161. Available from: http://www.aidsinfo.nih.gov/ContentFiles/ AdultandAdolescentGL.pdf Accessed Feb 26, 2010.

5. Hammer SM, Eron JJ Jr, Reiss P, et al. Antiretroviral treatment of adult HIV infection: 2008 recommendations of the International AIDS Society-USA panel. JAMA. 2008;300:555-570.

6. Gazzard BG, Anderson J, Babiker A, et al. British HIV Association Guidelines for the treatment of HIV-1-infected adults with antiretroviral therapy 2008. HIV Med. 2008;9:563-608.

7. Gardner EM, Hullsiek KH, Telzak EE, et al. Antiretroviral medication adherence and class-specific resistance in a large prospective clinical trial. AIDS. 2010;24:395-403.

8. Hirsch MS, Gunthard HF, Schapiro JM, et al. Antiretroviral drug resistance testing in adult HIV-1 infection: 2008 recommendations of an International AIDS Society-USA panel. Clin Infect Dis. 2008;47: 266-285.

9. Pauwels R. New non-nucleoside reverse transcriptase inhibitors (NNRTIs) in development for the treatment of HIV infections. Curr Opin Pharmacol. 2004;4:437-446.

10. Das K, Clark AD Jr, Lewi PJ, et al. Roles of conformational and positional adaptability in structure-based design of TMC125-R165335 (etravirine) and related non-nucleoside reverse transcriptase inhibitors that are highly potent and effective against wild-type and drug-resistant HIV-1 variants. $J$ Med Chem. 2004;47:2550-2560.

11. Andries K, Azijn H, Thielemans T, et al. TMC125, a novel next-generation nonnucleoside reverse transcriptase inhibitor active against nonnucleoside reverse transcriptase inhibitor-resistant human immunodeficiency virus type 1. Antimicrob Agents Chemother. 2004;48: 4680-4686.

12. De Corte BL. From 4,5,6,7-tetrahydro-5-methylimidazo[4,5,1-jk](1,4) benzodiazepin-2 $(1 \mathrm{H})$-one (TIBO) to etravirine (TMC125): fifteen years of research on non-nucleoside inhibitors of HIV-1 reverse transcriptase. J Med Chem. 2005;48:1689-1696.

13. European Medicines Agency. CHMP Assessment Report for Intelence. 2008. Available from: http://www.emea.europa.eu/humandocs/PDFs/ EPAR/intelence/H-900-en6.pdf Accessed Mar 1, 2010. 
14. Scholler-Gyure M, Kakuda TN, Raoof A, De Smedt G, Hoetelmans RM. Clinical pharmacokinetics and pharmacodynamics of etravirine. Clin Pharmacokinet. 2009;48:561-574.

15. Kakuda TN, Scholler-Gyure M, Workman C, et al. Single- and multipledose pharmacokinetics of etravirine administered as two different formulations in HIV-1-infected patients. Antivir Ther. 2008;13:655-661.

16. Scholler-Gyure M, Boffito M, Pozniak AL, et al. Effects of different meal compositions and fasted state on the oral bioavailability of etravirine. Pharmacotherapy. 2008;28:1215-1222.

17. Scholler-Gyure M, Kakuda TN, De Smedt G, et al. A pharmacokinetic study of etravirine (TMC125) co-administered with ranitidine and omeprazole in HIV-negative volunteers. Br J Clin Pharmacol. 2008;66: 508-516.

18. Kakuda TN, Scholler-Gyure M, Peeters M, et al. Pharmacokinetics of etravirine are not affected by sex, age, race, use of enfuvirtide or treatment duration in HIV-1 infected patients [abstract P34]. 9th International Workshop on Clinical Pharmacology of HIV Therapy. New Orleans, LA; 2008 Apr 7-9.

19. Scholler-Gyure M, Kakuda TN, De Smedt G, et al. Pharmacokinetics of TMC125 in HIV-1 negative volunteers with mild to moderate hepatic impairment [abstract A-1428]. 47th Interscience Conference on Antimicrobial Agents and Chemotherapy. Chicago, IL; 2007 Sep 17-20.

20. Aboud M, Castelino S, Back D, Kulasegaram R. Etravirine plasma levels in a patient with decompensated liver disease. AIDS. 2009;23: 1293-1295.

21. Giguere P, la Porte C, Zhang G, Cameron B. Pharmacokinetics of darunavir, etravirine and raltegravir in an HIV-infected patient on haemodialysis. AIDS. 2009;23:740-742.

22. Scholler-Gyure M, Woodfall B, De Marez T, et al. Pharmacokinetics of TMC125 with atazanavir and atazanavir/ritonavir [abstract P278]. 8th International Congress on Drug Therapy in HIV Infection. Glasgow; 2007 Nov 12-16.

23. Scholler-Gyure M, Woodfall B, Bollen S, et al. Pharmacokinetics of amprenavir and TMC125 in HIV-infected volunteers receiving TMC125 with fosamprenavir/ritonavir [abstract A-0370]. 46th Interscience Conference on Antimicrobial Agents and Chemotherapy. San Francisco, CA; 2006 Sep 27-30.

24. Scholler-Gyure M, Kraft M, Hoetelmans R, et al. Significant decrease in TMC125 exposures when co-administered with tipranavir (boosted with ritonavir) in healthy subjects [abstract 583]. 13th Conference on Retroviruses and Opportunistic Infections. Denver, CO; 2006 Feb 5-9.

25. Boffito M, Jackson A, Lamorde M, et al. Pharmacokinetics and safety of etravirine administered once or twice daily after 2 weeks treatment With efavirenz in healthy volunteers. J Acquir Immune Defic Syndr. 2009;52:222-227.

26. Kakuda TN, Scholler-Gyure M, De Smedt G, et al. Assessment of the steady-state pharmacokinetic interaction between etravirine administered as two different formulations and tenofovir disoproxil fumarate in healthy volunteers. HIV Med. 2009;10:173-181.

27. Scholler-Gyure M, Hoetelmans R, Bollen S, et al. No significant interaction between TMC125 and didanosine in healthy volunteers [abstract 29]. 6th International Workshop on Clinical Pharmacology of HIV Therapy. Quebec; 2005 Apr 28-30.

28. Davis J, Scholler-Gyure M, Kakuda TN, et al. An open, randomized, two-period, crossover study in two cohorts to investigate the effect of steady-state TMC125 (etravirine) and the combination of TMC125/ darunavir/ritonavir on the steady-state pharmacokinetics of oral maraviroc in healthy subjects [abstract P4.3/02]. 11th European AIDS Conference. Madrid; 2007 Oct 24-27.

29. Anderson MS, Kakuda TN, Hanley W, et al. Minimal pharmacokinetic interaction between the human immunodeficiency virus nonnucleoside reverse transcriptase inhibitor etravirine and the integrase inhibitor raltegravir in healthy subjects. Antimicrob Agents Chemother. 2008;52:4228-4232.

30. Menard A, Solas C, Mokthari S, et al. Etravirine-raltegravir, a marked interaction in HIV-1-infected patients: about four cases. AIDS. 2009;27: $869-871$.
31. Barrail-Tran A, Yazdanpanah Y, Fagard C, et al. Lack of interaction between etravirine and raltegravir plus darunavir/ritonavir when combined in treatment experienced patients: a substudy of the ANRS 139 TRIO trial [abstract 606]. 17th Conference on Retroviruses and Opportunistic Infections. San Francisco, CA; 2010 Feb 16-20.

32. Scholler-Gyure M, Debroye C, Woodfall B. Pharmacokinetic interaction between TMC125 and clarithromycin [abstract P1351]. 44th Annual Meeting, Infectious Diseases Society of America. Toronto; 2006 Oct 12-15.

33. Scholler-Gyure M, Kakuda TN, De Smedt G, et al. Pharmacokinetic interaction between non-nucleoside reverse transcriptase inhibitor (NNRTI) TMC125 and atorvastatin in HIV-negative volunteers [abstract WEPEA 106]. 4th IAS Conference on HIV Pathogenesis, Treatment and Prevention. Sydney; 2007 Jul 22-25.

34. Scholler-Gyure M, Debroye C, Vyncke V, et al. Effect of TMC125 on sildenafil pharmacokinetics. 7th International Workshop of Clinical Pharmacology of HIV Therapy. Lisbon; 2006 Apr 20-22.

35. Tibotec Inc. Intelence (etravirine) Product Monograph. Raritan. NJ, USA; 2010 Feb.

36. Scholler-Gyure M, Kakuda TN, Van Solingen R, et al. No clinically relevant effect of etravirine (ETR: TMC125) on digoxin pharmacokinetics in HIV seronegative volunteers [abstract P22]. 9th International Workshop on Clinical Pharmacology of HIV Therapy. New Orleans, LA; 2008 Apr 7-9.

37. Scholler-Gyure M, Woodfall B, Debroye C, et al. Pharmacokinetic interaction between TMC125 and rifabutin [abstract 963]. 44th Annual Meeting, Infectious Diseases Society of America. Toronto; 2006 Oct 12-15.

38. Scholler-Gyure M, Kakuda TN, Stevens T, et al. Effect of etravirine on cytochrome $\mathrm{P} 450$ isozymes assessed by Cooperstown $5+1$ cocktail [abstract A-955]. 48th Interscience Conference on Antimicrobial Agents and Chemotherapy. Washington, DC; 2008 Oct 25-28.

39. Vingerhoets J, Azijn H, Fransen E, et al. TMC125 displays a high genetic barrier to the development of resistance: evidence from in vitro selection experiments. J Virol. 2005;79:12773-12782.

40. Lazzarin A, Campbell T, Clotet B, et al. Efficacy and safety of TMC125 (etravirine) in treatment-experienced HIV-1-infected patients in DUET-2: 24-week results from a randomised, double-blind, placebocontrolled trial. Lancet. 2007;370:39-48.

41. Madruga JV, Cahn P, Grinsztejn B, et al. Efficacy and safety of TMC125 (etravirine) in treatment-experienced HIV-1-infected patients in DUET-1: 24-week results from a randomised, double-blind, placebocontrolled trial. Lancet. 2007;370:29-38.

42. Vingerhoets J, Tambuyzer L, Azijn H, et al. Resistance profile of etravirine: combined analysis of baseline genotypic and phenotypic data from the randomized, controlled Phase III clinical studies. AIDS. 2010;24:503-514.

43. Cotte L, Trabaud MA, Tardy JC, et al. Prediction of the virological response to etravirine in clinical practice: comparison of three genotype algorithms. J Med Virol. 2009;81:672-677.

44. Poveda E, Garrido C, de Mendoza C, et al. Prevalence of etravirine (TMC-125) resistance mutations in HIV-infected patients with prior experience of non-nucleoside reverse transcriptase inhibitors. J Antimicrob Chemother. 2007;60:1409-1410.

45. Llibre JM, Santos JR, Puig T, et al. Prevalence of etravirine-associated mutations in clinical samples with resistance to nevirapine and efavirenz. J Antimicrob Chemother. 2008;62:909-913.

46. Lapadula G, Calabresi A, Castelnuovo F, et al. Prevalence and risk factors for etravirine resistance among patients failing on non-nucleoside reverse transcriptase inhibitors. Antivir Ther. 2008;13:601-605.

47. Manosuthi W, Butler DM, Chantratita W, Sukasem C, Richman DD, Smith DM. Mutations associated with etravirine resistance among patients infected with HIV-1 subtype CRF01_AE and failing first-line nevirapine and efavirenz-based regimens. Antivir Ther. 2009;14:A155 [abstract 32].

48. MacArthur RD, Hullsiek KH, Peng G, et al. Failing therapy with efavirenz results in significantly fewer mutations limiting etravirine than failing therapy with nevirapine: on-treatment analyses from the CPCRA FIRST Study [abstract 129]. XVII international HIV Drug Resistance Workshop. Sitges, Spain; 2008 Jun 10-14. 
49. ClinicalTrials.gov. Etravirine clinical studies. Accessed Mar 10, 2010.

50. Gruzdev B, Rakhmanova A, Doubovskaya E, et al. A randomized, doubleblind, placebo-controlled trial of TMC125 as 7-day monotherapy in antiretroviral naive, HIV-1 infected subjects. AIDS. 2003;17: 2487-2494.

51. Gazzard BG, Pozniak AL, Rosenbaum W, et al. An open-label assessment of TMC 125 - a new, next-generation NNRTI, for 7 days in HIV-1 infected individuals with NNRTI resistance. AIDS. 2003;17:F49-F54.

52. Montaner J, Yeni P, Clumeck NN, et al. Safety, tolerability, and preliminary efficacy of 48 weeks of etravirine therapy in a phase IIb dose-ranging study involving treatment-experienced patients with HIV-1 infection. Clin Infect Dis. 2008;47:969-978.

53. Nadler JP, Berger DS, Blick G, et al. Efficacy and safety of etravirine (TMC125) in patients with highly resistant HIV-1: primary 24-week analysis. AIDS. 2007;21:F1-F10.

54. Cohen CJ, Berger DS, Blick G, et al. Efficacy and safety of etravirine (TMC125) in treatment-experienced HIV-1-infected patients: 48-week results of a phase IIb trial. AIDS. 2009;23:423-426.

55. Ruxrungtham K, Pedro RJ, Latiff GH, et al. Impact of reverse transcriptase resistance on the efficacy of TMC125 (etravirine) with two nucleoside reverse transcriptase inhibitors in protease inhibitor-naive, nonnucleoside reverse transcriptase inhibitor-experienced patients: study TMC125-C227. HIV Med. 2008;9:883-896.

56. Yazdanpanah Y, Fagard C, Descamps D, et al. High rate of virologic suppression with raltegravir plus etravirine and darunavir/ritonavir among treatment-experienced patients infected with multidrug-resistant HIV: results of the ANRS 139 TRIO trial. Clin Infect Dis. 2009;49: 1441-1449.

57. Mills A, Cahn P, Molina JM, Nijs S, Vingerhoets J, Witek J. Etravirine demonstrates durable efficacy in treatment-experienced patients in the DUET trials: pooled 96-week results [abstract MOPEB036]. 5th IAS Conference on HIV Pathogenesis, Treatment and Prevention. Capetown, South Africa; 2009 Jul 19-22.
58. Katlama C, Haubrich R, Lalezari J, et al. Efficacy and safety of etravirine in treatment-experienced, HIV-1 patients: pooled 48 week analysis of two randomized, controlled trials. AIDS. 2009;23: 2289-2300.

59. Girard PM, Grinsztejn B, Rachline A, Nijs S, Witek J. Etravirine demonstrates a favourable safety and tolerability profile: pooled 96-week results from the phase III DUET trials [abstract PE7.3/5]. 12th European AIDS Conference. Cologne; 2009 Nov 11-14.

60. Peeters M, Janssen K, Kakuda TN, et al. Etravirine has no effect on QT and corrected QT interval in HIV-negative volunteers. Ann Pharmacother. 2008;42:757-765.

61. Furco A, Gosrani B, Nicholas S, et al. Successful use of darunavir, etravirine, enfuvirtide and tenofovir/emtricitabine in pregnant woman with multiclass HIV resistance. AIDS. 2009;23:434-435.

62. Izurieta P, Kakuda TN, Clark AD Jr, Feys C, Witek J. Safety and pharmacokinetics of etravirine in pregnant HIV-infected women [abstract PE4.1/6]. 12th European AIDS Conference. Cologne; 2009 Nov 11-14.

63. Peeters K, Viala M, Gilet H, et al. Health-related quality of life (HROL) as measured by the Functional Assessment of HIV (FAHI) questionnaire in treatment-experienced HIV-1-infected patients: 24-week results from the pooled DUET trials. 11th European AIDS Conference. Madrid; Oct 24-27 2007

64. Staszewski S, Morales-Ramirez J, Tashima KT, et al. Efavirenz plus zidovudine and lamivudine, efavirenz plus indinavir, and indinavir plus zidovudine and lamivudine in the treatment of HIV-1 infection in adults. Study 006 Team. N Engl J Med. 1999;341: 1865-1873.
Virus Adaptation and Treatment

\section{Publish your work in this journal}

Virus Adaptation and Treatment is an international, peer-reviewed open access journal focusing on the study of virology, viral adaptation and the development and use of antiviral drugs and vaccines to achieve improved outcomes in infection control and treatment. The journal welcomes original research, basic science, clinical \& epidemiological

\section{Dovepress}

studies, reviews \& evaluations, expert opinion and commentary, case reports and extended reports. The manuscript management system is completely online and includes a very quick and fair peer-review system, which is all easy to use. Visit http://www.dovepress.com/ testimonials.php to read real quotes from published authors. 